\title{
Review of: "Colo-colic intussusception secondary to colon lipoma: A case report"
}

\author{
Ishna Sharma
}

Potential competing interests: The author(s) declared that no potential competing interests exist.

This article is well-written and provides a nice reminder that the etiology for intussusception in adult patients is usually some sort of mass causing the intussusception, and that one example of such a mass can be a lipoma. 